\title{
Spectropolarimetric Properties of a Gallium Nanoparticle Layer on a Sapphire Substrate
}

\author{
Prashant Raman, ${ }^{1}$ Kirk Fuller, ${ }^{2}$ and Don A. Gregory ${ }^{1}$ \\ ${ }^{1}$ Department of Physics, University of Alabama in Huntsville, 301 Sparkman Drive, Huntsville, AL 35899, USA \\ ${ }^{2}$ Earth System Science Center, University of Alabama in Huntsville, 320 Sparkman Drive, Huntsville, AL 35805, USA \\ Correspondence should be addressed to Prashant Raman; prashant.raman@gmail.com
}

Received 29 May 2014; Revised 19 September 2014; Accepted 22 September 2014; Published 2 November 2014

Academic Editor: Gunjan Agarwal

Copyright (c) 2014 Prashant Raman et al. This is an open access article distributed under the Creative Commons Attribution License, which permits unrestricted use, distribution, and reproduction in any medium, provided the original work is properly cited.

Gallium nanoparticles (Ga NPs) are currently the subject of vigorous research as possible substrates in surface-enhanced Raman scattering (SERS) experiments in the ultraviolet spectral domain. Verification of any comprehensive model of the interaction of electromagnetic radiation with Ga NPs requires that complete polarimetric measurements be made. These spectropolarimetric properties can be obtained using a Mueller matrix spectropolarimeter (MMSP). The position of localized surface plasmon resonances (LSPRs) and spectral depolarization data of Ga NPs in the 300 to $1100 \mathrm{~nm}$ spectral region are presented. Spectral depolarization data may be of value in creating a better understanding of how light couples to individual nanoparticles, as well as the role played by interparticle coupling and the connection to phenomena such as SERS.

\section{Introduction}

The optical properties of metal nanoparticles have caused them to be considered in recent years for applications ranging from sensing to modifying the emission and absorption spectra of a variety of materials. Typical metals considered for such nanoparticle studies have traditionally been $\mathrm{Au}$ and Ag, both of which have LSPR peaks in the near UV, visible, or IR region. Ga NPs, on the other hand, can be tuned into the deep UV as a result of the high plasma frequency of Ga. Additionally, the LSPR for Ga NPs have broad tunability, stability across a wide temperature range, excellent plasmon resiliency when oxidized, and simplicity of deposition even at room temperature [1-3].

Ellipsometric measurements determine the amplitude ratio $(\psi)$ and phase shift $(\Delta)$ between the parallel and perpendicular components of the polarization state. The complex refractive index and thickness of the thin film can be calculated from the spectral dependence of $\psi$ and $\Delta[4$, 5]. The peaks in the imaginary component of the refractive index (corresponding to absorption) identify the positions and strengths of the LSPRs. Ellipsometric measurements can be performed over a range of wavelengths and incident angles in order to achieve additional insight and a better estimate of the optical constants. However, conventional ellipsometry cannot measure polarization parameters such as circular retardance, circular diattenuation, and depolarization.

Recently, a Mueller matrix spectropolarimeter (MMSP) was developed at the University of Alabama in Huntsville (UAH) with the aim of studying the polarization properties of various optical/electrooptical elements, materials, microparticle deposits, and so forth. The MMSP configured in the reflection mode can parallel the operation of a conventional ellipsometer and simultaneously provide additional polarization information about the sample. The development and performance of the MMSP have been reported and measured data presented on the polarization characteristics of particulates and the MMSP's potential as a bioaerosol detector [6-8].

\section{The MMSP}

Figure 1 is a schematic of the broadband Mueller matrix polarimeter configured for measurement in reflection mode while Figure 2 is a photograph of the MMSP in that configuration. The design is based on the dual rotating retarder 


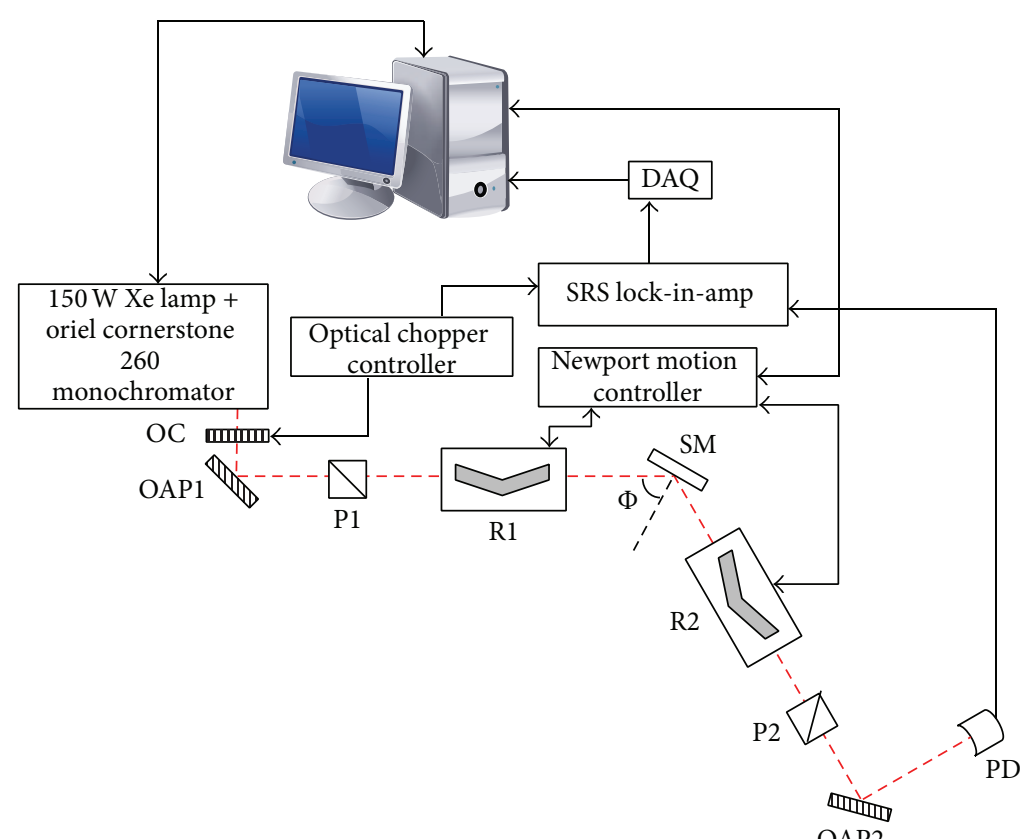

OC: optical chopper

OAP1/2: off-axis parabolic mirror

$\mathrm{P} 1 / 2$ : rochon polarizer
R1/2: Fresnel-rhomb retarder

PD: Si photodiode

DAQ: data acquisition board

FIGURE 1: Schematic of the broadband Mueller matrix polarimeter.

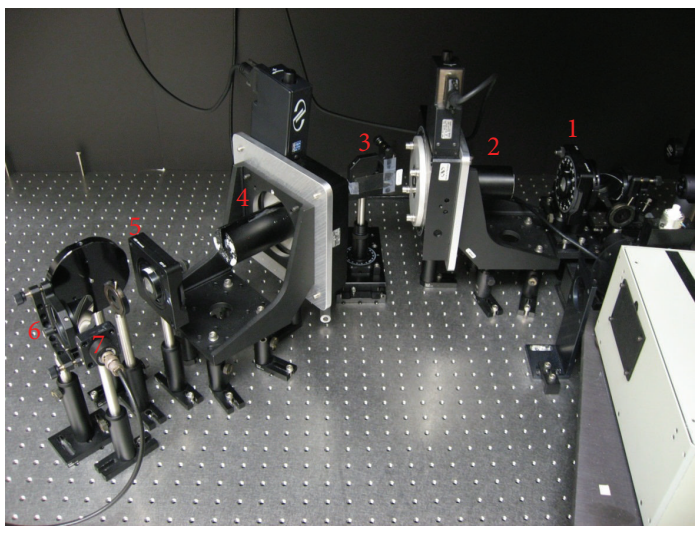
(1) PSG polarizer
(5) PSA polarizer
(2) PSG retarder
(6) OAP mirror
(3) Sample holder
(7) Si photodiode
(4) PSA retarder

FIGURE 2: Photograph of MMSP in reflection mode.

polarimeter proposed by Azzam [9], which is capable of providing all 16 elements of the Mueller matrix. In this design, both the polarization state generator (PSG) and polarization state analyzer (PSA) are comprised of a pair of polarizers (fixed in orientation) and quarter wave retarders (each mounted on a rotation stage). The polarimeter is comprised of four major subsystems, namely, the optical subsystem, the motion-control subsystem, the electronic subsystem, and the software subsystem. Details of the instrument design and operation may be found in the literature [7].

The UAH MMSP operates seamlessly in the $300 \mathrm{~nm}$ to $1100 \mathrm{~nm}$ wavelength region at a resolution of $5 \mathrm{~nm}$. The system can be configured in different incarnations: transmission, reflection, back-scattering, and so forth. In this report, results are given for the MMSP in the reflection mode (MMSP-R) such that light is incident on the sample at a grazing $\left(20^{\circ}\right)$ 


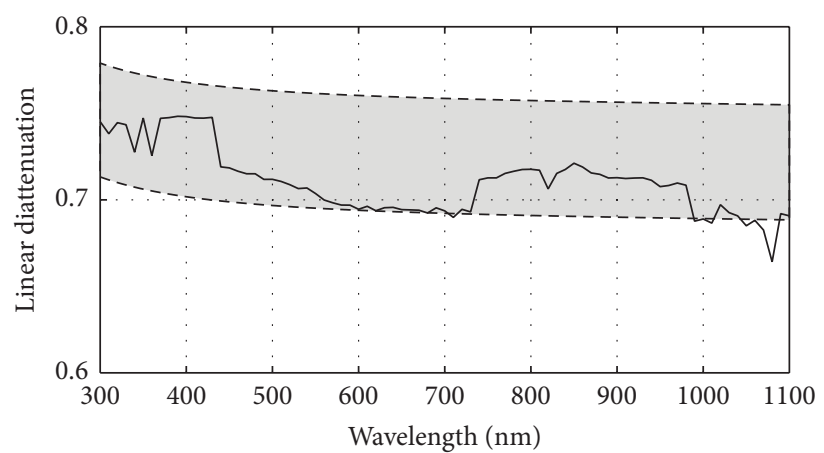

Figure 3: Diattenuation due to reflection from microscope slide (theoretical and as measured by MMSP).

angle and the specular reflection from the sample is analyzed by the PSA.

In order to validate the performance of the MMSP$\mathrm{R}$, the reflection from a borosilicate microscope slide was measured. The linear diattenuation produced by the dielectric surface can be calculated from the spectral dependence of the refractive index over the desired wavelength region and the Fresnel equations [10]. Figure 3 is a plot of the theoretical and measured values of the linear diattenuation of the microscope slide. The shaded area corresponds to the spread in the theoretically calculated diattenuation values over the range of incident angles from $69^{\circ}$ to $71^{\circ}$. This is done to account for possible misalignment of the slide in the sample holder. The solid black line corresponds to the measured values of diattenuation which are mostly within the range of expected misalignment.

\section{Ga Nanoparticle Layer}

Researchers at Duke University (DU) prepared the Ga nanoparticle layers, which were deposited via high vacuum molecular beam epitaxy (HVMBE) and the refractive index was determined in situ using conventional ellipsometry [3]. The three samples investigated in this paper were labeled A5, A6, and A8. Each is a result of a different deposition time and hence they have different size distributions. Thus, the LSPR peaks occur at different photon energies. Figure 4 shows scanning electron microscope (SEM) images of samples A5 and $\mathrm{A} 8$ and their corresponding size distribution histograms. Note that the SEM image of A5 is for a magnification of $150000 x$ while the SEM image of A8 is for a magnification of $100000 \mathrm{x}$ and that the latter has a bimodal size distribution. Particle size statistics and an SEM image were not available for sample A6.

Figure 5 shows atomic force microscope (AFM) images of the three samples A5, A6, and A8. It is clear from the images that A6 has a size distribution with a mean particle radius smaller than that in sample A5. Table 1 lists the mean radius and LSPR peak for the three samples. Since sample A8 has a bimodal size distribution, two mean radii are given.

The ellipsometric measurements performed by DU reveal that the longitudinal mode (or lower energy mode) of the LSPR peak occurs in sample A5 at $324.6 \mathrm{~nm}$, in sample A6 at $291.0 \mathrm{~nm}$, and in sample A8 at $626.2 \mathrm{~nm}$. The much longer wavelength of the LSPR for A8 corresponds to the transverse mode in the $70 \mathrm{~nm}$ sized nanoparticles. The $16 \mathrm{~nm}$ size nanoparticles in the same sample are expected to have a LSPR peak in the deep UV, beyond the measuring capability of DU's in situ spectroscopic ellipsometer. The transverse modes (or higher energy modes) are blue-shifted and also beyond the measuring capability of the ellipsometer.

\section{Measurements on Ga Nanoparticle Layer}

The MMSP in reflection mode was assembled for measurements at grazing $\left(20^{\circ}\right)$ incidence so as to allow comparison with measurements made at DU. The measurements performed at DU focused on the complex refractive index, $n+i k$, of Ga films on sapphire wafers in order to characterize LSPRs associated with the nanostructure of the $\mathrm{Ga}$. Such resonances are indicated by peaks in the absorption index $k$.

Depolarization is expected to be strongly angle dependent and a more pronounced depolarization may have occurred at a different angle of incidence. However, the LSPR peaks are expected to be only weakly dependent on the angle of incidence for the particle geometries considered. Also, the MMSP does not easily lend itself to a change in configuration. Hence, the measurements presented here are limited to the $20^{\circ}$ angle.

4.1. Mueller Matrix and Polarization Properties. Figure 6 shows the spectral plots (ranging from $250 \mathrm{~nm}$ to $1100 \mathrm{~nm}$ ) of all 16 elements of the Mueller matrix of samples A5, A6, and A8, represented by green, blue, and red colors, respectively. The standard deviation in the measurements is about the width of the line. The difference between the three samples is apparent in the symmetric elements $m_{01}\left(=m_{10}\right)$ and $m_{22}$ $\left(=m_{33}\right)$ as well as the antisymmetric elements $m_{23}\left(=-m_{32}\right)$ of the Mueller matrix. This can be attributed to the particle size difference between the samples. Diattenuation due to the sample appears in element $m_{01}$ and retardance appears in element $m_{23}$ of the Mueller matrix. Any antisymmetry between the symmetric elements and symmetry between the antisymmetric elements indicate the presence of depolarization in the measured sample.

Any nonsingular Mueller matrix $(M)$ can be decomposed as $M=M_{\text {Dep }} M_{\text {Ret }} M_{\text {Diat }}$, where $M_{\text {Dep }}$ is the Mueller matrix of a pure depolarizer, $M_{\text {Ret }}$ is the Mueller matrix of a pure 

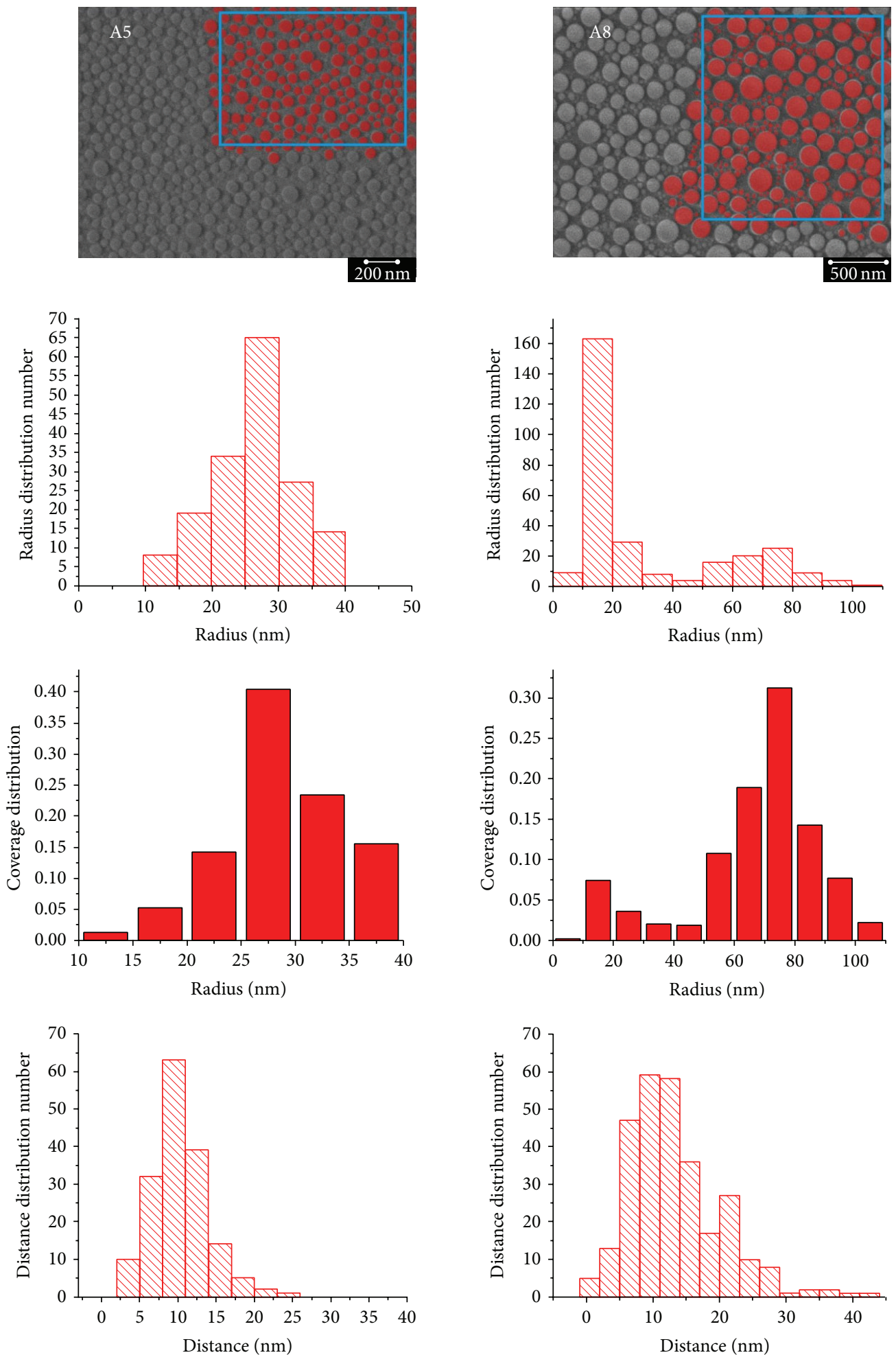

FIGURE 4: SEM images of samples A5 and A8 with corresponding size distribution histograms. 


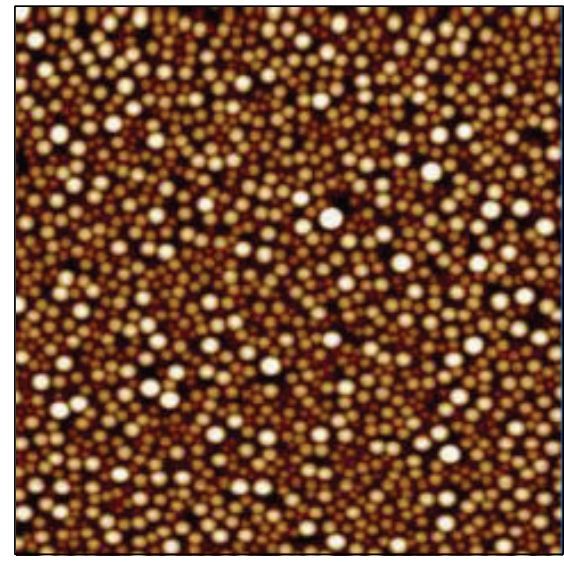

A6

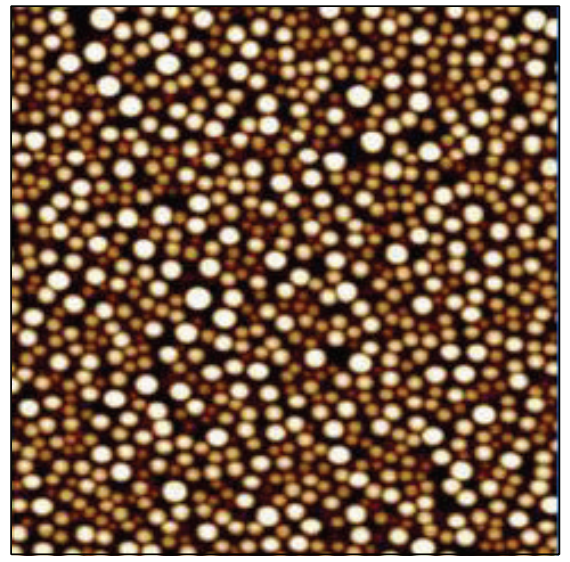

A5

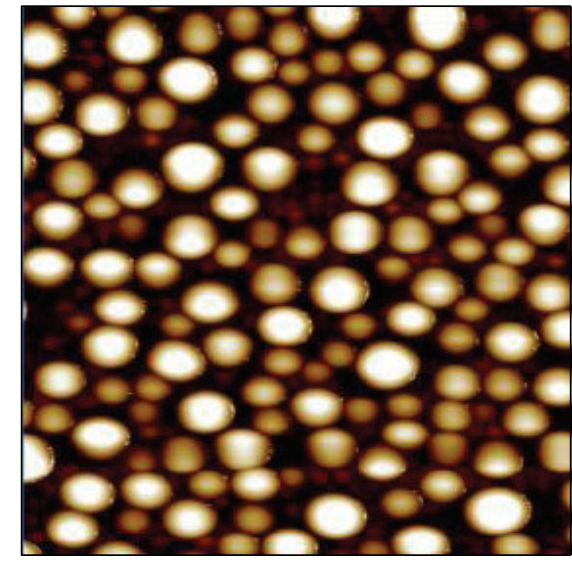

A8

Figure 5: AFM images of samples A6, A5, and A8.
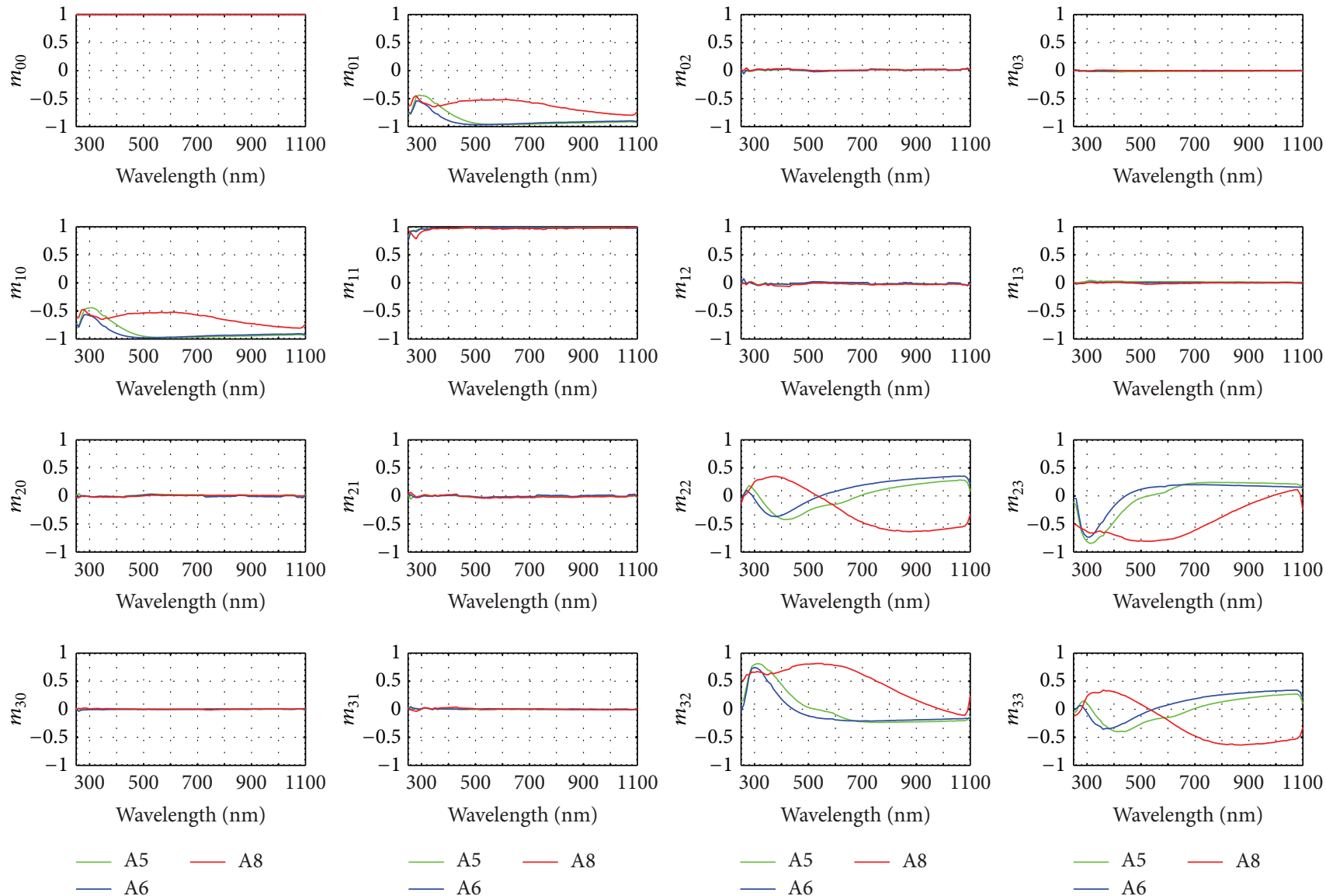

- A5 - A8

$-\mathrm{A} 5-\mathrm{A} 8$

- A5

- A8

Figure 6: Mueller matrix plots of samples A5, A6, and A8.

TABLE 1: Ga sample characteristics.

\begin{tabular}{cccccc}
\hline & $\begin{array}{c}\text { Mean radius } \\
(\mathrm{nm})\end{array}$ & $\begin{array}{c}\text { LSPR peak } \\
(\mathrm{nm})\end{array}$ & $\begin{array}{c}\text { Density } \\
\left(\# / \mu \mathrm{m}^{2}\right)\end{array}$ & $\begin{array}{c}\text { Coverage } \\
(\%)\end{array}$ & $\begin{array}{c}\text { Neighbor distance } \\
(\mathrm{nm})\end{array}$ \\
\hline A6 & $<26$ & 291.0 & - & - & - \\
A5 & 26 & 324.6 & 131 & 43.7 & 10 \\
A8 & $16 \& 70$ & 626.2 & 104 & 51.4 & 13 \\
\hline
\end{tabular}



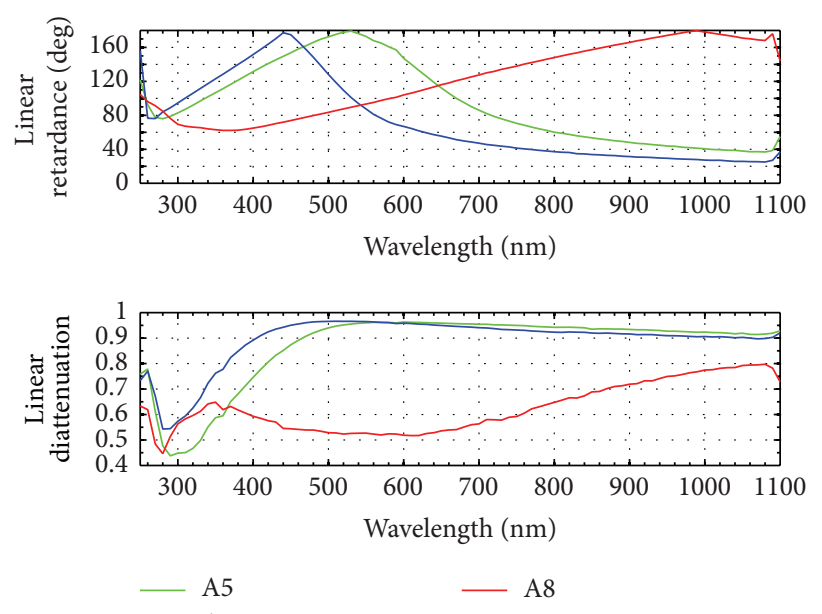

$-\mathrm{A} 8$

Figure 7: Plot of the linear retardance and linear diattenuation of samples A5, A6, and A8.

retarder, and $M_{\text {Diat }}$ is the Mueller matrix of a pure diattenuator. The decomposed Mueller matrices and polarization properties are calculated using standard methods [11]. Figure 7 is a plot of linear retardance and linear diattenuation for samples A5, A6, and A8. Again, the standard deviation is about the width of the line.

The dielectric sapphire substrate itself contributes little to the linear diattenuation since this data is taken in reflection and the surface is substantially covered. The Ga nanoparticle layer is expected to dominate the overall linear retardance for the same reason. The diattenuation of bare sapphire was measured to be about 0.87 (in reflection, 70-degree incident angle) over the entire wavelength range of interest and the retardance was measured to be less than $5^{\circ}$ (essentially zero) over the same range.

4.2. The Pseudodielectric Function [4]. The in situ ellipsometric data from DU is used to calculate the ratio of the complex reflection coefficients $\left(R_{p} / R_{s}\right)$ of the sample for the two incident orthogonal states of polarization. This ratio can be written as $[4,12]$

$$
\rho=\frac{R_{p}}{R_{s}}=\tan \psi \cdot\left(e^{i \Delta}\right), \quad\left(0 \leq \psi \leq 90^{\circ} ; 0 \leq \Delta<360^{\circ}\right),
$$

where $\tan \psi=\left|R_{p}\right| /\left|R_{s}\right|$ is the relative amplitude attenuation and $\Delta=\arg \left(R_{p}\right)-\arg \left(R_{s}\right)$ is the differential phase shift between the $p$ and $s$ linearly polarized components upon reflection. The pseudodielectric function $\langle\varepsilon\rangle$, which is related to the above $\rho$, can be calculated in real time, as the measurement occurs, using

$$
\langle\varepsilon\rangle=(n+i k)^{2}=n_{o}^{2} \sin ^{2} \varphi\left[1+\left(\frac{1-\rho}{1+\rho}\right)^{2} \tan ^{2} \varphi\right],
$$

where $n_{o}$ is the refractive index of the incident medium and $\varphi$ is the angle of incidence.

Figure 8 is a plot of the pseudoextinction coefficient $k$ versus wavelength for samples A5, A6, and A8, as determined by the in situ spectroscopic ellipsometer at DU (labeled DUKE) and the ex situ Mueller matrix ellipsometric measurements determined here (labeled UAH). Samples A5, A6, and A8 have LSPR peaks occurring at $360 \mathrm{~nm}, 330 \mathrm{~nm}$, and $730 \mathrm{~nm}$, respectively, as measured by the MMSP. Since the above defined pseudodielectric coefficient assumes a semi-infinite substrate with nanoparticles, negative values for $k$ measured by DU and UAH may be attributed to reflection from the back surface of the substrate of the samples considered herein. The red-shifts observed in the ex situ MMSP spectra are attributed to the samples having been coated with a fluorescent dye (for SERS studies, which is what the samples were originally prepared for), as well as oxidation due to aging. Such redshifts are predicted from theories for the interaction of coated nanoparticles with light [3]. The difference in the peak amplitudes between the DU and UAH measurement can be attributed to the data being collected using two different techniques with no common calibration between the instruments.

4.3. The Depolarization Index. Conventional ellipsometers cannot measure the full set of polarization properties, including circular retardance, circular diattenuation, and depolarization, which is needed to completely describe the interaction of electromagnetic radiation with metallic nanoparticle systems. For the samples considered here, a significant amount of depolarization was observed. The depolarization index is defined as [13]

$$
\operatorname{Dep}(M)=1-\frac{\sqrt{\left(\sum_{i, j} m_{i j}^{2}\right)-m_{00}^{2}}}{\sqrt{3} m_{00}},
$$

where $M$ is the Mueller matrix and $m_{i j}$ are the Mueller matrix elements. Figure 9 is a plot of the depolarization index for samples A5, A6, and A8. The standard deviation again is roughly equal to the width of the line. The lack of structure in the curve for sample A8 in the figure is due to the Mueller matrix being decomposed into three Mueller matrices representing retardance, diattenuation, and 


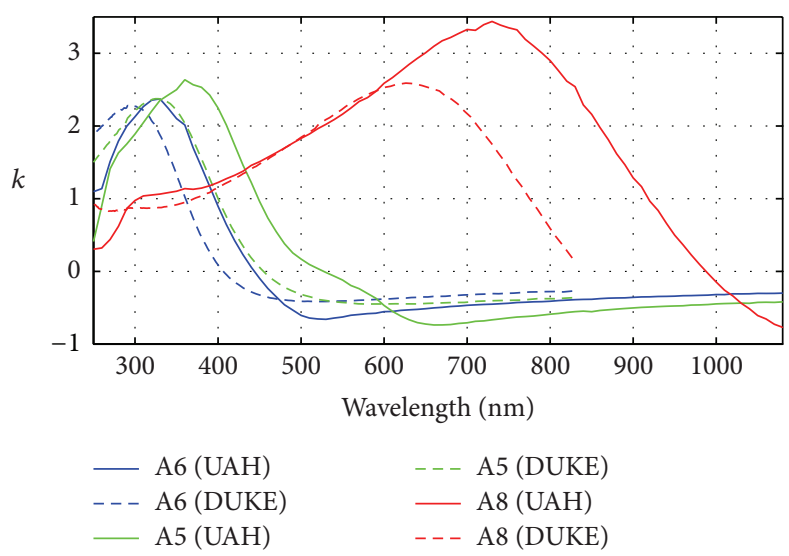

FIGURE 8: Imaginary part of the refractive index for samples A5, A6, and A8.

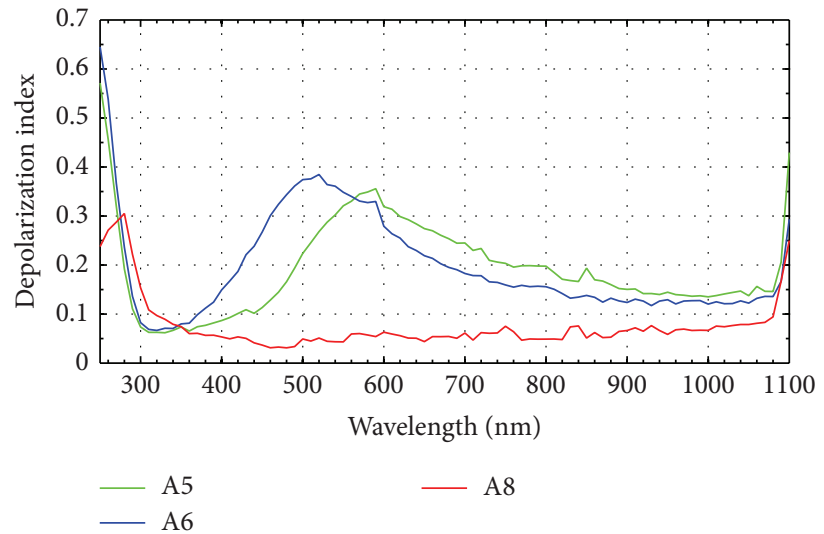

FIgURE 9: Plot of depolarization index of samples A5, A6, and A8.

depolarization. The depolarization index is calculated from the Mueller matrix representing depolarization. This matrix for A8 (not shown) was very close to an identity matrix while the other two samples displayed significant spectral features.

Depolarization has not previously been quantified for nanoparticle systems such as the ones under consideration here. An important finding of the work conducted thus far is a strong, sample-dependent signature in the depolarization index. The peaks in the depolarization curve for samples A5 and A6 occur at $590 \mathrm{~nm}$ and $520 \mathrm{~nm}$, respectively. The more interesting feature is the minima in the depolarization curves for these samples. The minima in depolarization index for sample A5 occurs at $360 \mathrm{~nm}$, coinciding with the measured LSPR peak of $360 \mathrm{~nm}$. The minima in depolarization index for sample A6 occurs at $320 \mathrm{~nm}$, close to the measured LSPR peak of $330 \mathrm{~nm}$. For very different sample geometries, it is observed that the two parameters peak at about the same wavelength [14]. The perceived correlation between the depolarization minima and the LSPR peaks is not fully understood and is the subject of ongoing investigations. It should be noted that the plots in Figure 9 range from $250 \mathrm{~nm}$ to $1100 \mathrm{~nm}$. Any features seen below $300 \mathrm{~nm}$ and near $1100 \mathrm{~nm}$ cannot be deemed reliable due to the low SNR associated with instrument cutoff.

\section{Conclusion}

In this investigation, previously unknown polarization features, particularly depolarization, have been extracted from spectropolarimetric measurements using the MMSP. The Mueller matrix ellipsometric measurements of Ga nanoparticle layers reveal LSPR peaks that are red-shifted compared to the in situ spectroscopic ellipsometric measurements, which is likely due to the (intended and unintended) modification of the sample surfaces. There appears to be an unexpected correlation between the LSPR peak spectral positions and the spectral positions of the minima in the depolarization index.

\section{Conflict of Interests}

The authors declare that there is no conflict of interests regarding the publication of this paper.

\section{Acknowledgments}

The authors wish to thank Art Lompado for his assistance during the development phase of the MMSP and for helpful technical input. Henry Everitt and Yang Yang provided the samples and the results of their measurements performed 
at DU. The polarimetric instrumentation was made possible by the Army's Defense University Research Instrumentation Program (DURIP). Thanks also are due to John Christy and Mike Newchurch for their cooperation and support.

\section{References}

[1] P. Wu, Plasmonic gallium nanoparticles-attributes and applications [Ph.D. dissertation], Duke University, 2009.

[2] P. C. Wu, C. G. Khoury, T.-H. Kim et al., "Demonstration of surface-enhanced Raman scattering by tunable, plasmonic gallium nanoparticles," Journal of the American Chemical Society, vol. 131, no. 34, pp. 12032-12033, 2009.

[3] P. C. Wu, T.-H. Kim, A. S. Brown, M. Losurdo, G. Bruno, and H. O. Everitt, "Real-time plasmon resonance tuning of liquid Ga nanoparticles by in situ spectroscopic ellipsometry," Applied Physics Letters, vol. 90, no. 10, Article ID 103119, 2007.

[4] R. M. A. Azzam, "Ellipsometry," in Handbook of Optics, M. Bass, C. DeCusatis, J. Enoch et al., Eds., vol. 1, chapter 16, McGrawHill, New York, NY, USA, 3rd edition, 2009.

[5] R. M. A. Azzam and N. M. Bashara, Ellipsometry and Polarized Light, North-Holland, Amsterdam, The Netherlands, 1989.

[6] P. Raman, Spectropolarimetric characterization of light scattering materials [Ph.D. dissertation], University of Alabama in Huntsville, 2012.

[7] P. Raman, "Broadband (UV-VIS-NIR) Mueller matrix polarimeter," in Polarization Science and Remote Sensing V, vol. 8160 of Proceedings of SPIE, 2011.

[8] P. Raman, K. Fuller, and D. Gregory, "Polarimetric discrimination of atmospheric particulate matter," in Polarization: Measurement, Analysis, and Remote Sensing X, vol. 8364 of Proceedings of SPIE.

[9] R. M. A. Azzam, "Photopolarimetric measurement of the Mueller matrix by Fourier analysis of a single detected signal," Optics Letters, vol. 2, no. 6, pp. 148-150, 1978.

[10] http://www.us.schott.com/advanced_optics/english/download/ schott-optical-glass-collection-datasheets-june-2014-us.pdf.

[11] S.-Y. Lu and R. A. Chipman, "Interpretation of Mueller matrices based on polar decomposition," Journal of the Optical Society of America A: Optics and Image Science, and Vision, vol. 13, no. 5, pp. 1106-1113, 1996.

[12] D. H. Goldstein and E. Collett, Polarized Light, CRC Press, Boca Raton, Fla, USA, 2003.

[13] R. A. Chipman, "Polarimetry," in Handbook of Optics, M. Bass, Ed., McGraw-Hill, New York, NY, USA, 1995.

[14] G. F. Walsh, C. Forestiere, and L. Dal Negro, "Plasmonenhanced depolarization of reflected light from arrays of nanoparticle dimers," Optics Express, vol. 19, no. 21, pp. 21081-21090, 2011. 

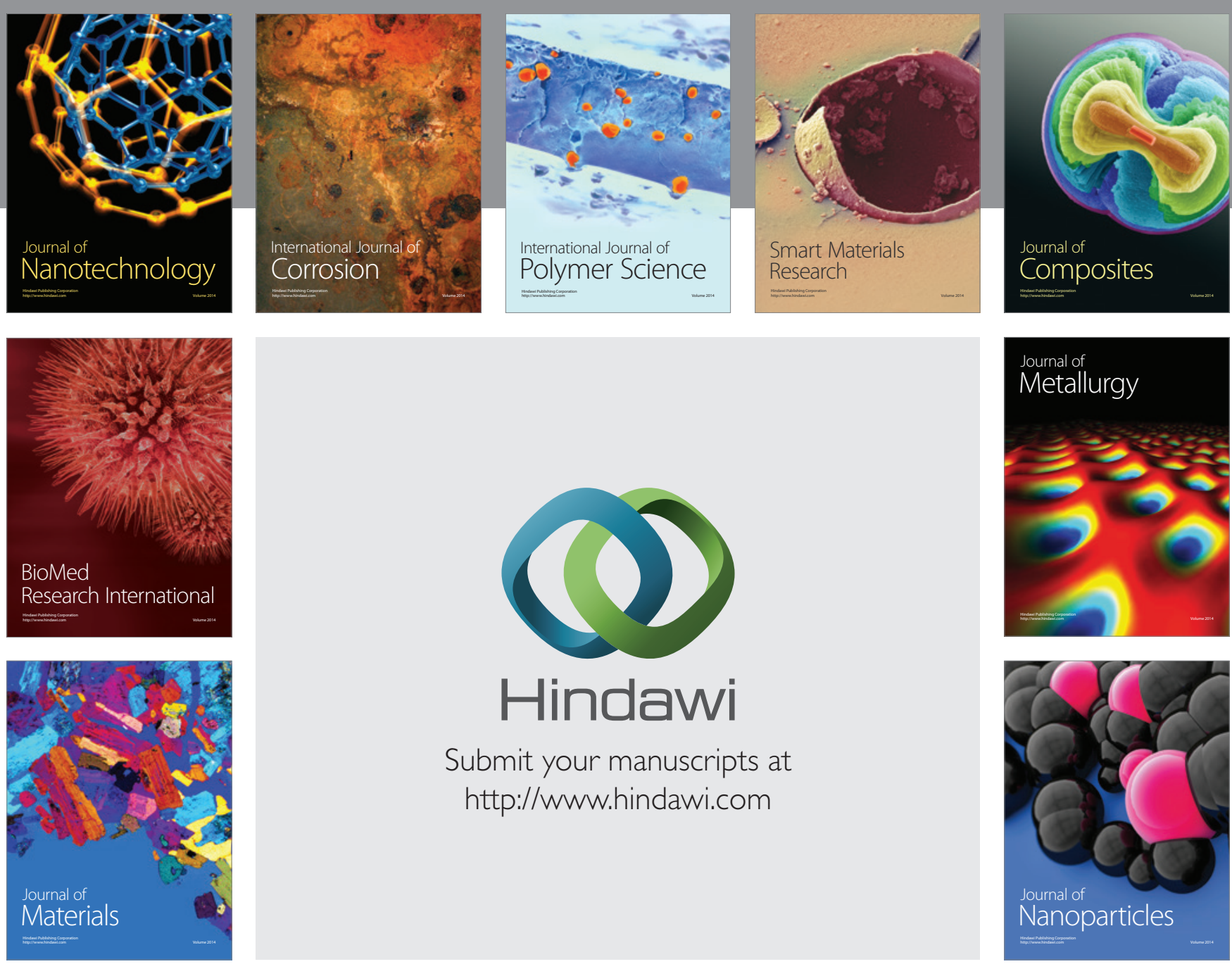

Submit your manuscripts at http://www.hindawi.com
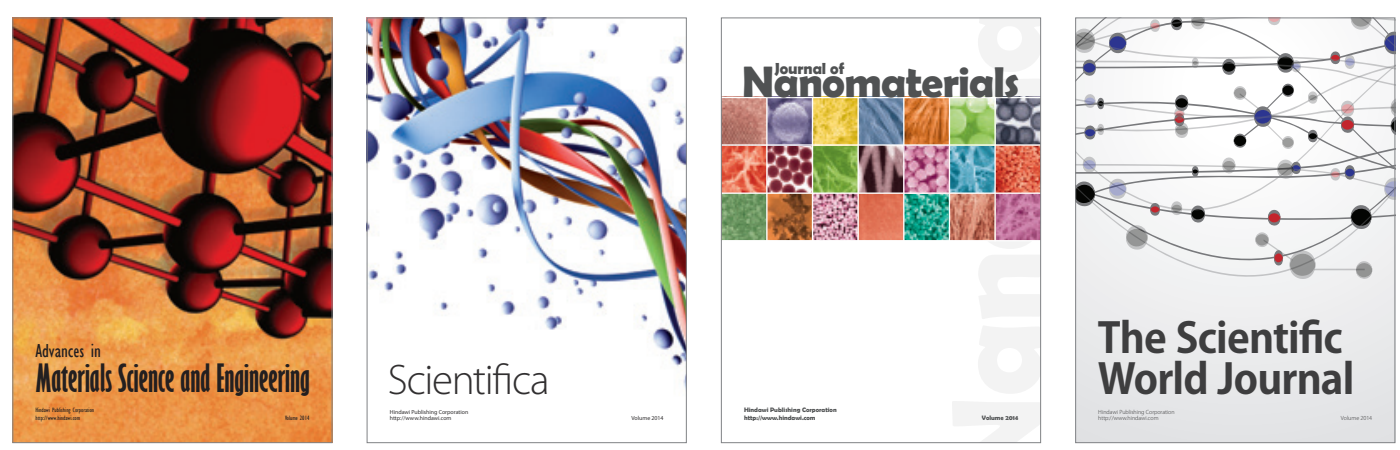

\section{The Scientific World Journal}
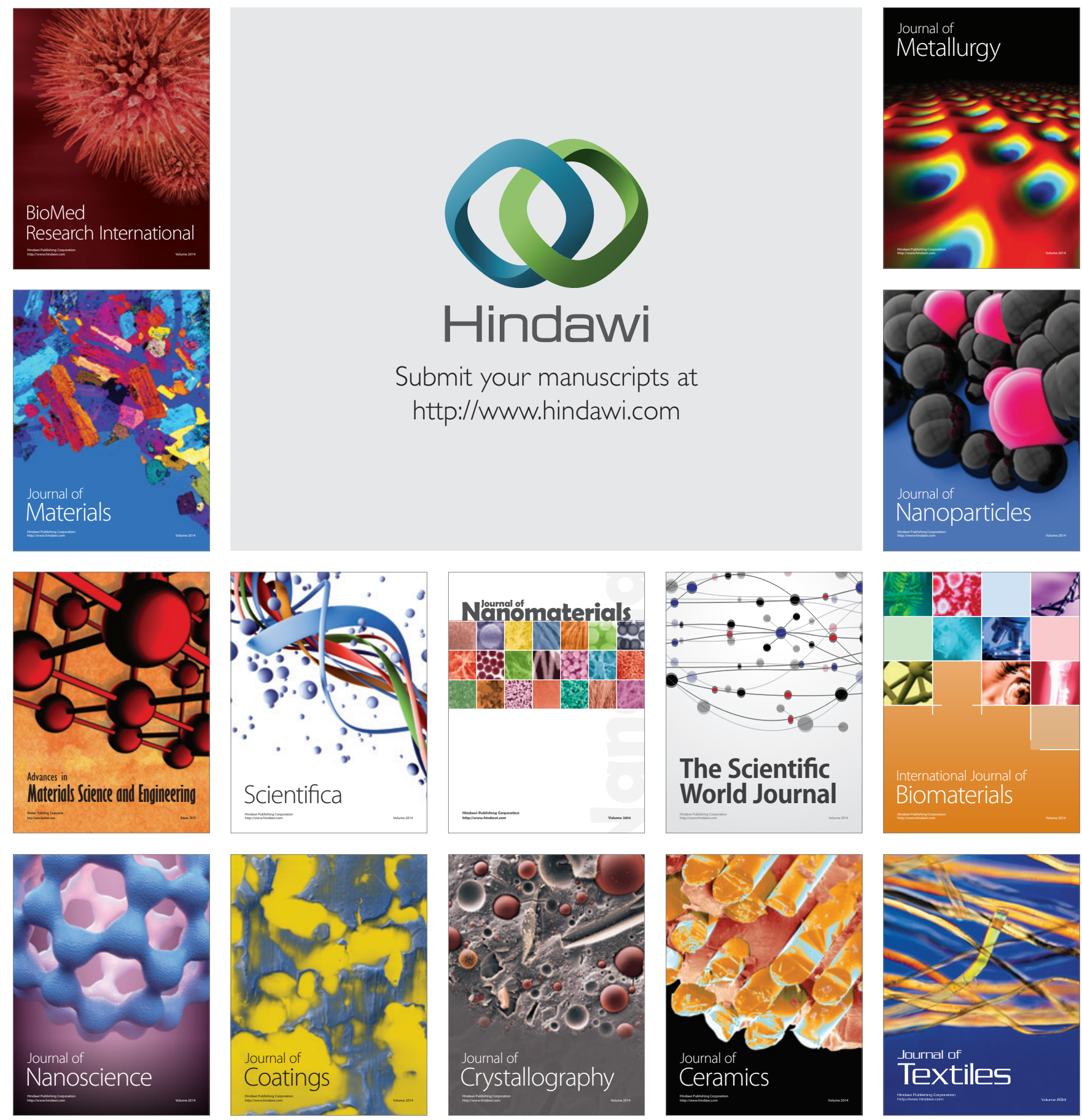